\title{
Campo elétrico pulsado
}

\author{
Pulsed electric field
}

\section{Mirella Lima Binoti ${ }^{I}$ Afonso Mota Ramos ${ }^{\text {* }}$ Luciano José Quintão Teixeira ${ }^{\text {II }}$ Paulo Cesar Stringheta Valéria Rodrigues de Paula Minim ${ }^{\mathrm{I}}$ Mônica Ribeiro Pirozi ${ }^{\mathrm{I}}$}

\section{- REVISÃO BIBLIOGRÁFICA -}

\section{RESUMO}

Campos elétricos pulsados de alta intensidade (CEPAI) constituem um método não-térmico de conservação para alimentos em substituição à pasteurização tradicional. Em comparação ao processamento térmico, os CEPAI, além de serem eficientes na eliminação de micro-organismos e na inativação de enzimas, também minimizam as perdas de sabor, cor, textura, nutrientes e componentes termolábeis dos alimentos. $O$ objetivo desse trabalho foi levantar dados bibliográficos atuais sobre o tema CEPAI, abordando: definição do processo, instalação experimental, efeito da ruptura dielétrica do alimento, efeito sobre micro-organismo, enzimas e constituintes dos alimentos. Os dados encontrados na literatura apontam o tratamento por CEPAI como alternativa promissora para a conservação de alimentos, podendo ser utilizado como tecnologia única ou como complemento aos processamentos térmicos.

Palavras-chave: campo elétrico pulsado de alta intensidade, micro-organismos, características nutricionais $e$ sensoriais.

\section{ABSTRACT}

Pulsed electric fields of high intensity (HIPEF) is a non-thermal food conservation to replace traditional thermal pasteurization. Compared to thermal processing, the HIPEF as well as being effective in removing microorganisms and inactivate enzymes also minimize the loss of flavor, color, texture, nutrients and labile components of food. The aim of this work was to gather bibliographic data current HIPEF on the subject, covering: experimental setup, effects of dielectric breakdown of food, effect on microorganisms, enzymes and constituents of food. The data found in the literature indicate treatment of
HIPEF as a promising alternative for food conservation and it could be used as a single technology or in addition to thermal processing.

Key words: high-intensity pulsed electric field, microorganisms, nutrition and sensory characteristics

\section{INTRODUÇÃO}

Campo elétrico pulsado de alta intensidade (CEPAI) é uma das tecnologias não térmicas de processamento mínimo de interesse para os cientistas e para a indústria alimentícia, sendo um método novo e alternativo para conservação de alimentos líquidos. Além disso, é uma alternativa promissora aos tratamentos térmicos tradicionais, que tem apresentado bons resultados, não apenas por possibilitar a destruição de micro-organismos e a inativação de enzimas, mas também por manter o sabor, a cor, a textura, as vitaminas e componentes funcionais termolábeis dos alimentos (CÓRTES et al., 2008). Portanto, a aplicação dessa tecnologia não térmica de conservação de alimentos satisfaz ao consumidor que está cada vez mais exigente em relação aos produtos que consomem, ou seja, em sua busca por alimentos processados mais similares aos in natura, e que apresentem elevada qualidade nutricional e sensorial (RIENER et al., 2008).

\footnotetext{
'Departamento de Ciência e Tecnologia de Alimentos, Universidade Federal de Viçosa (UFV), 36570-000, Viçosa, MG, Brasil. E-mail: amramos@ufv.br. *Autor para correspondência.

"Departamento de Ciência e Tecnologia de Alimentos, Universidade Federal do Espírito Santo (UFES), Campus Alegre, Alegre, ES, Brasil.
} 
A proposta deste trabalho é realizar uma revisão de literatura acerca do tema campo elétrico pulsado na conservação de alimentos, com vistas a explicitar definição do processo, instalação experimental, efeito da ruptura dielétrica do alimento, efeito sobre micro-organismo, enzimas e constituintes dos alimentos.

Campo Elétrico Pulsado de Alta Intensidade (CEPAI)

A tecnologia consiste em submeter o produto a campos de alta intensidade (na ordem de 5 a 55 kilovolts por centímetro $-\mathrm{kV} \mathrm{cm}{ }^{-1}$ ) com pulsos elétricos de curta duração ( $\mathrm{ms}$ ou $\mu \mathrm{s})$, repetidos muitas vezes (constituindo o número de pulsos) com a finalidade de inativar enzimas e destruir microorganismos (PETTIT et al., 2002; SOLIVA-FORTUNY et al., 2009).

Quando o alimento é submetido a uma diferença de potencial $(\mathrm{V})$, produz aquecimento devido a sua resistência elétrica intrínseca. Este processo é conhecido como aquecimento ôhmico, e causa perdas de alguns nutrientes termolábeis, e por isso há o interesse no desenvolvimento de processos que não causem aquecimento e consequentemente provoquem alterações mínimas nos alimentos. Para minimizar o efeito Joule provocado pelo tratamento ôhmico, e diminuir o aquecimento do produto se desenvolveu o tratamento via CEPAI, que utiliza pulsos elétricos de curta duração. Assim, utilizam-se os efeitos elétricos e não os térmicos para conservar os alimentos. Atualmente faz-se a distinção entre campo elétrico com finalidade de aquecer os alimentos (tratamento ôhmico) e CEPAI usado para submeter o alimento a uma "pasteurização" não térmica (TEIXEIRA, 2008).

\section{Instalação experimental}

Nas últimas décadas, inúmeros estudos têm sido desenvolvidos para aperfeiçoar os equipamentos, para esclarecer sobre os mecanismos de atuação do CEPAI sobre micro-organismos e enzimas, e também para estabelecer os parâmetros de tratamento para diferentes produtos alimentícios.

A unidade de processamento de alimentos típica para campos elétricos é um sistema simples, composto por uma fonte geradora de pulsos elétricos (fonte de alta voltagem) e uma câmara de tratamento (WAN, 2009). Dependendo do sistema utilizado, podese incluir alguns parâmetros de tratamento típico de campo elétrico pulsado, como intensidade de campo de 15 a $50 \mathrm{kV} \mathrm{cm}^{-1}$, comprimento de pulso de 1 a $5 \mu \mathrm{s} \mathrm{e}$ freqüência de pulso de 200 e $400 \mathrm{~Hz}$ (WAN, 2009).

O sistema gerador de pulsos elétricos é composto por um gerador de alta tensão e um gerador de pulso, um banco de capacitores, resistências elétricas, um osciloscópio para medir a intensidade dos pulsos elétricos gerados e outros componentes eletrônicos comuns a vários sistemas elétricos. Os pulsos elétricos formados podem possuir forma (onda) quadrada, exponencial, oscilatória e bipolar (BARBOSA-CÁNOVAS et al., 1999). É possível observar a forma do pulso plotando-se um gráfico de intensidade do campo elétrico versus tempo. No pulso exponencial, o campo elétrico atinge um valor máximo e depois decai de forma exponencial. No pulso quadrado o campo elétrico se mantem em um valor máximo por certo período para depois cair abruptamente formando um quadrado no gráfico. Já os pulsos oscilantes e bipolares são obtidos por circuitos particulares capazes de produzir estas formas de pulsos (PETTIT et al., 2002).

As câmaras de tratamento, as quais podem ser estáticas ou contínuas, possuem dois eletrodos entre os quais passa o alimento a ser tratado, além de um conjunto de dispositivos de monitoramento e controle (SOLIVA-FORTUNY et al., 2009). As câmaras estáticas tratam os alimentos em bateladas e são utilizadas com eficiência em atividades de pesquisa, já as contínuas proporcionam um fluxo contínuo e são mais adequadas aos processos industriais. Com a finalidade de maximizar a eficiência das câmaras, vários destes equipamentos surgiram nas ultimas décadas, os quais são planejados para possibilitar um tratamento homogêneo, ter máxima eficiência na inativação de enzimas, na destruição de micro-organismos; minimizarem possíveis reações eletroquímicas e evitar que ocorra ruptura dielétrica no alimento enquanto estiver sendo submetido ao tratamento de CEPAI (TEIXEIRA, 2008). Uma nova concepção da câmara de tratamento foi descrita por ALKHAFAJI \& FARID (2007), apresentando dois eletrodos inoxidáveis de malha de aço que permitem que o campo elétrico de alta intensidade tenha apenas um limitado aumento na temperatura do líquido, de modo que esses eletrodos apresentam-se também associados à limitadas incrustações, evitando assim, a ruptura dielétrica do alimento.

\section{Ruptura dielétrica do alimento}

A ruptura dielétrica consiste numa descarga elétrica entre dois eletrodos da câmara, um processo análogo a um relâmpago. Esta descarga de energia pode catalisar reações indesejáveis nos alimentos, ou em casos mais graves, a explosão da câmara de tratamento. A ocorrência desse fenômeno está relacionada à condutividade elétrica do alimento, assim, alimentos sólidos, apresentam maior probabilidade de ocorrência 
da ruptura dielétrica. Por isso, líquidos homogêneos, além de serem facilmente bombeados nos equipamentos utilizados para o tratamento, permitem um fluxo continuo, e apresentam menor probabilidade de ocorrência da ruptura dielétrica (TEIXEIRA, 2008). Esse processo ocorre quando o campo elétrico ultrapassa a força dielétrica do alimento, o qual se caracteriza por correntes elétricas elevadas fluindo em canais estreitos; evolução de bolhas de gás; formações de incrustações nos eletrodos e aumento da pressão acompanhado por um som explosivo (ZHANG et al., 1995).

\section{Efeito sobre micro-organismo}

O processamento por CEPAI é uma alternativa de conservação capaz de destruir microorganismos deteriorantes e patogênicos sem afetar significativamente as propriedades sensoriais e nutricionais de vários alimentos (ELEZ-MARTÍNEZ et al., 2006). No entanto, o nível de inativação microbiana pelo CEPAI depende de muitos fatores que são críticos para a eficácia do tratamento.

A exposição de uma célula biológica ao CEPAI conduz a um fenômeno de permeabilização de membrana, chamado de eletroporação. Esse mecanismo ainda não é totalmente compreendido (LEBOVKA et al., 2004; ARONSSON et al., 2005), porém, sabe-se que se caracteriza por provocar desestabilização da bicamada lipídica e das proteínas de membrana, levando a formação de poros, e ocorre quando a membrana excede um valor potencial crítico de campo elétrico. A intensidade do pulso elétrico é diretamente proporcional à destruição microbiana, ou seja, quanto maior for a intensidade do pulso (acima do potencial de transmembrana crítico) maior será o grau de destruição. O potencial transmembrana natural da célula é de aproximadamente $1 \mathrm{~V}$, de modo que se a intensidade do pulso for superior a esse limite ocorre, provavelmente, aumento da permeabilidade da membrana com formação de poros e eventual ruptura celular (KNORR et al., 2001).

O processo de permeabilização celular é um processo dinâmico e pode ser reversível ou irreversível dependendo da intensidade do tratamento. A ruptura pode ser reversível, se o campo elétrico for abaixo de certo valor crítico e por um período de tempo curto. Porém, se forem ultrapassados certos valores de intensidade de campo e de tempo de tratamento, o processo torna-se irreversível, ocorrendo perda de material celular e morte da célula (KNORR et al., 2001).

Muitas hipóteses têm sido propostas para tentar elucidar se a iniciação dos poros se dá na parte protéica ou na parte lipídica da bicamada da membrana. O ponto de consenso entre os cientistas é que o mecanismo de ação do CEPAI nos micro-organismos está relacionado a mudanças na membrana citoplasmática (RAMOS et al., 2006). O apoio à hipótese de inativação microbiana devido à permeabilização da membrana pelo CEPAI foi relatado por ARONSSON et al. (2005), quando os autores demonstraram que o tratamento com CEPAI em Escherichia coli, Listeria innocua e Saccharomyces cerevisiae aumentou a captação do corante de iodeto fluorescente (PI) e vazamento de compostos intracelulares, embora não tenham sido observadas evidencias de ruptura de membrana, apenas formação de poros em células.

A permeabilização da membrana está envolvida na inativação de bactérias por CEPAI, mas a extensão, e eventualmente, a natureza dos danos de membrana dependem das características do produto a ser tratado e/ou fatores do meio $(\mathrm{pH}$, presença de antimicrobianos e compostos iônicos; condutividade elétrica, força iônica e atividade de água) (GARCIA et al., 2007). Também a intensidade e duração do pulso, o tempo e a temperatura de tratamento, o tipo do eletrodo e formas do pulso (onda) interferem na inativação microbiana (OTUNOLA et al., 2008; AZERÊDO et al., 2008).

Quanto maior for a diferença entre a condutividade elétrica do meio e o citoplasma microbiano, maior será o dano à estrutura da membrana, devido ao aumento no fluxo de substâncias iônicas através dela. A taxa de destruição de micro-organismos aumenta com o decréscimo da condutividade elétrica. Quanto menor a atividade de água maior é a resistência microbiana aos diferentes tratamentos ou agentes de inativação. $\mathrm{O}$ pH atua como fator de estresse adicional, ao contrário da presença de cátions bivalentes que parecem exercer forte efeito sobre a redução da letalidade por pulso elétrico.

GARCIA et al. (2005) relataram que $\boldsymbol{L}$. monocytogenes foi sensível ao tratamento por campos elétricos em pH 4,0; mas apresentou maior resistência em pH 7,0. Em contraste, E. coli O157: H7 e Salmonella senftenberg foram sensíveis ao tratamento em $\mathrm{pH} 7,0$; e resistente em $\mathrm{pH} 4,0$.

Uma redução maior que cinco ciclos logarítmicos (UFC mL $\mathrm{mL}^{-1}$ ) de população de Escherichia coli O157: H7, Salmonella enteritidis e Listeria monocytogenes, foi conseguida com tratamento de CEPAI (em suco de melão $-35 \mathrm{kV} \mathrm{cm}^{-1}$ por $1709 \mu \mathrm{s} \mathrm{a}$ $193 \mathrm{~Hz}$ e $4 \mu \mathrm{s}$, e em suco de melancia $-35 \mathrm{kV} \mathrm{cm}^{-1}$ por $1682 \mu \mathrm{s}$ a $193 \mathrm{~Hz}$ e $4 \mu \mathrm{s})$ em combinação com ácido cítrico $(0.5-2.0 \%, \mathrm{p} / \mathrm{v})$ ou com óleo de casca de canela 0.05-0.30\%, p/v) (MOSQUEDA-MELGAR et al., 2008). A destruição de Escherichia Coli 8739 foi estudada em extrato hidrossolúvel de soja, verificando-se que o tratamento de CEPAI a $41 \mathrm{kV} \mathrm{cm}^{-1}$ por $54 \mu \mathrm{s}$, ocasionou 
uma redução maior que cinco ciclos logarítmicos (UFC $\mathrm{mL}^{-1}$ ) (LI \& ZHANG, 2004). Os autores também observaram que o efeito do tratamento na destruição foi mais eficiente com os aumentos de intensidade do campo elétrico e do tempo total do tratamento.

Estudos indicam que o CEPAI é um tratamento útil para a redução de Pseudomonas, a maior causa de deterioração bacteriana do leite. Os tratamentos com CEPAI resultaram em vários graus de destruição microbiana, dependendo da temperatura associada ao tratamento e também dos tipos de microorganismos presentes no meio (CRAVEN et al., 2008). O maior grau destruição de Lactobacillus plantarum foi observado com a maior intensidade de campo e menor tempo de tratamento. Quando a temperatura do processo foi elevada para $55^{\circ} \mathrm{C}$ as reduções aumentaram, atingindo um decréscimo de 0,5 ciclos logarítmicos (SAMPEDRO et al., 2007).

As características dos micro-organismos alvo (espécie e cepa, morfologia, concentração e fase de crescimento), também influenciam no grau de letalidade do tratamento. As bactérias Gram-positivas são mais resistentes ao tratamento por pulso elétrico do que as Gram-negativas; os fungos normalmente são mais sensíveis do que bactérias devido ao maior tamanho, embora pareçam ser mais resistentes do que as células Gram-negativas aos pulsos de menor intensidade. Em geral, células nas fases logarítmicas são mais sensíveis ao estresse do que aquelas na fase lag ou estacionária (AZERÊDO; OLIVEIRA\& FARO, 2008).

Estudos têm mostrado diferenças significativas na eficácia entre pulsos mono e bipolar para a inativação bactérias Gram-positiva (Bacillus cereus, Listeria monocytogenes NCTC 11994) e Gramnegativa (Escherichia coli NCTC 9001, E. coli O157: H7)(BEVERIDGE etal., 2005; EVRENDILEK \&ZHANG 2005). A presença de uma parede celular possuindo grossa camada de mucopeptídio nas bactérias Grampositivas, em contraste com a membrana de bactérias Gram-negativas, pode contribuir para uma maior resistência ao tratamento com CEPAI (WAN et al., 2009).

É amplamente relatado que o tratamento com CEPAI é ineficaz para a destruição de esporos de bactérias (WAN et al., 2009). Esses esporos parecem resistir bem à ação do CEPAI, por serem menores e mais circulares, o que confere certa proteção, sendo mais difícil destruí-los do que bactérias, leveduras e fungos filamentosos. Os pulsos elétricos não induzem a germinação, embora possa ser induzida por outros tratamentos, de forma que os pulsos elétricos venham a ser utilizados em seguida para destruir as células recém germinadas (TEIXEIRA, 2008).
O efeito da destruição pode ser aumentado ao aplicar a tecnologia de CEPAI em combinação com outros fatores de estresse, como a presença de compostos antimicrobianos tais como nisina e ácidos orgânicos, o aumento da atividade de água, calor, $\mathrm{pH}$ e tratamentos térmicos mais brandos. Todos eles têm um efeito sinérgico na destruição, porém mais estudos são necessários para compreender os mecanismos envolvidos, mais especificamente, quando os efeitos sobre os esporos estão em questão(DEVLIEGHERE et al., 2004).

\section{Efeito sobre enzimas}

Os mecanismos envolvidos na inativação de enzimas ainda não são totalmente esclarecidos (ZHAO \& YANG, 2009), porém sabe-se que proteínas com atividade enzimática podem ser inativadas sob algumas condições de tratamento com CEPAI. Os resultados de vários estudos indicaram que a intensidade do campo, duração e o número de pulsos, são as principais variáveis que afetam a inativação enzimática (HO et al., 1997).

Quando uma enzima é submetida a um campo elétrico, ocorre sua exposição a uma força elétrica devido aos seus grupos carregados em posições diferentes. Por causa desses grupos surgem forças elétricas perturbadoras que podem levar a mudança em sua conformação estrutural provocando a desnaturação. Outros autores sugerem que a inativação pode ocorrer devido a oxidação de alguns componentes da enzima como grupos sulfidrilas (ZHONG et al., 2005).

ZHONG et al. (2005) relataram que a conformação da peroxidase (POD) mudou após o tratamento com CEPAI, sendo a redução aumentada com o aumento dos parâmetros do tratamento. Os resultados caracterizaram a mudança de conformação de uma $\alpha$-hélice. Resultados semelhantes foram observados por ZHONG et al. (2007).

Estudos relatam a inativação de pepsina por CEPAI, correlacionando a inativação com a alteração da estrutura secundária (estrutura $\beta$-folha) (ZHAO \& YANG, 2009). Esses estudos têm relatado o efeito do CEPAI sobre a estrutura das enzimas, em particular a estrutura secundária tridimensional (ZHAO \& YANG, 2009). Portanto, as alterações na atividade enzimática podem ser resultados dessas modificações.

A inativação da lipoxigenase (LOX) de soja por CEPAI foi estudada por LI et al. (2008). A atividade residual da enzima diminuiu com o aumento do tempo de tratamento, intensidade, freqüência e comprimento de pulso. Já RIENER et al. (2008) conseguiram a inativação com um tempo menor, combinando tratamento de CEPAI com tratamento térmico (préaquecimento a $50^{\circ} \mathrm{C}$ ). 
Contudo, a maior ou menor resistência da enzima ao CEPAI dependerá do número de pontes de hidrogênio que ela possui, e da sua composição em aminoácidos que lhe conferirá maior ou menor hidrofobicidade, da presença de metais em sua estrutura e também do seu volume (BENDICHO et al., 2002a).

Efeito sobre constituintes dos alimentos

Embora a destruição de micro-organismos e enzimas por CEPAI venham sendo extensivamente estudadas, pouco se sabe sobre os efeitos dessa tecnologia sobre outros constituintes dos alimentos (AZERÊDO et al., 2008). Na atualidade, não apenas uma longa vida de prateleira, mas também outros aspectos de qualidade dos alimentos são importantes para o consumidor. Consequentemente, tecnologias de processos não térmicos têm sido alvos de pesquisas, buscando-se avaliar o potencial desses processamentos como métodos alternativos ao tratamento térmico convencional para eliminar ou minimizar a degradação da qualidade dos alimentos (ARONSSON et al., 2005).

O tratamento CEPAI, também pode ser proposto como tecnologia quando se deseja conservar a composição lípidica dos alimentos. Após o tratamento de CEPAI em suco de laranja, enriquecido com ácidos graxos poli insaturados ( $\omega-3$ e ácido oléico) não foram observadas alterações significativas $(\mathrm{P}<0,05)$ nos teores desses componentes (ZULUETA et al., 2007).

Muitos estudos têm relatado a vantagem da aplicação de CEPAI para a conservação de micronutrientes dos alimentos, pois se espera que as vitaminas termolábeis sejam conservadas, já que o pulso elétrico não constitui tratamento térmico. BENDICHO et al. (2002b) avaliaram o efeito do pulso elétrico sobre as vitaminas hidrossolúveis (riboflavina, tiamina e ácido ascórbico) e lipossolúveis (colecalciferol e tocoferol) do leite. Os autores relataram mudanças no conteúdo vitamínico, com exceção do ácido ascórbico (vitamina C) que apresentou maior retenção $(93,4 \%)$ no leite tratado por pulso elétrico do que o submetido à pasteurização. BAZHAL et al. (2001) tratando suco de laranja por pulso elétrico verificaram retenção de todas suas propriedades físicas e cerca de $97,5 \%$ de retenção da vitamina C. As reações de degradação da vitamina C são muitas vezes responsáveis por mudanças significativas de qualidade que ocorrem durante o armazenamento de alimentos, como a formação de compostos intermediários instáveis (ex.: furfural), e isso limita a vida útil do produto (ZULUETA et al., 2009).

Os efeitos do tratamento com CEPAI em micro elementos ou compostos bioativos presentes nos alimentos não têm sido sempre considerados (SOLIVAFORTUNY et al., 2009). A maioria dos estudos que relacionam o efeito do CEPAI sobre os componentes relacionados com a saúde estuda apenas as alterações no conteúdo de vitamina $\mathrm{C}$.

No entanto, ODRIOZOLA-SERRANO et al. (2009) avaliaram o efeito do processamento com CEPAI sobre o conteúdo de vitamina $\mathrm{C}$, teor de antocianinas e a capacidade antioxidante em suco de morango. Os resultados demonstraram que as amostras tratadas com pulso bipolar obtiveram $2,3 \%$ menor retenção de vitamina $\mathrm{C}$ quando comparado ao modo monopolar, porém, os conteúdos de antocianina foram maiores no tratamento com pulso bipolar. A capacidade antioxidante máxima do suco fresco também foi alcançada com pulsos bipolares. Esse estudo demonstra que os parâmetros do tratamento são importantes para promover uma maior ou menor conservação dos componentes dos alimentos.

O tratamento com CEPAI também têm sido investigado para modificar características de tecidos de frutas e hortaliças. Uvas Tempranillo foram submetidas a tratamento com CEPAI e os resultados mostraram que a permeabilização da casca da uva através da aplicação de um pré-tratamento com CEPAI em temperatura ambiente causa um incremento da intensidade de cor, teor de antocianinas e do conteúdo de polifenóis totais em relação ao controle (casca sem pré-tratamento) durante todo o processo de vinificação (LÓPEZ et al., 2008).

O CEPAI demonstra-se eficiente para a manutenção da cor dos alimentos, fato importante para a aceitação do consumidor. Suco de laranja tratado com CEPAI apresenta uma coloração mais semelhante ao suco de laranja não tratado, e menores índices de escurecimento quando comparado ao produto submetido à pasteurização térmica (CORTÉS et al., 2008). As coordenadas de cor também não se alteram em suco de cenoura (TEIXEIRA et al., 2008) e em sucos de frutas cítricas (grapefruit, limão, laranja, tangerina) (CSERHALMI et al., 2006) tratados com CEPAI.

Outra vantagem da tecnologia é provocar mínima ou nenhuma alteração nas características físicoquímicas dos alimentos. $\mathrm{O} \mathrm{pH},{ }^{\circ}$ Brix, condutividade elétrica, viscosidade, índice de escurecimento nãoenzimático (Nebi) e hidroxi-metil-furfural (HMF) de sucos de frutas cítricas (grapefruit, limão, laranja, tangerina) tratados com CEPAI, não se alteraram significativamente $(\mathrm{P}<0,05)$ (CSERHALMI et al., 2006). Resultados semelhantes foram obtidos por CORTÉS et al. (2008), onde o $\mathrm{pH}$ e o ${ }^{\circ}$ BRIX de suco de laranja tratado com CEPAI $\left(30 \mathrm{kV} \mathrm{cm}^{-1}, 100 \mu \mathrm{s}\right)$, não apresentaram alterações estatísticas significativas $(\mathrm{P}<0,01)$ 
imediatamente após o tratamento, quando comparados aos produtos frescos ou com os tratados com pasteurização térmica.

Contudo, para o sucesso de uma nova tecnologia é necessário que o produto tratado seja aceito pelo consumidor. NIELSEN et al. (2009) analisaram a atitude de consumidores frente a novas tecnologias (CEPAI e Processamento por Alta pressão) em 6 países (Eslovênia, Hungria, Sérvia, Eslováquia, Noruega e Dinamarca), por meio de um estudo com grupos de foco, utilizando um guia comum e dois produtos tratados com essa tecnologia (suco e um alimento infantil). Os resultados mostraram que os consumidores perceberam as principais vantagens do tratamento para o produto, as quais foram, ser mais parecido com o natural, melhoria do sabor e manutenção do valor nutritivo mais próximo ao original, enquanto que a principal desvantagem foi a falta de informações sobre as metodologias.

\section{CONSIDERAÇÕES FINAIS}

Os estudos sugerem que tratamento via CEPAI pode ser uma tecnologia viável para garantir a produção de alimentos seguros do ponto de vista microbiológico sem que afete substancialmente a qualidade nutricional e sensorial dos mesmos. É uma alternativa interessante para a indústria de alimentos, pois trata-se de um investimento interessante, uma vez que aumentaria o lucro, além de viabilizar a obtenção de alimentos mais saudáveis e com características mais naturais, propiciando maior satisfação do consumidor.

\section{REFERÊNCIAS}

ALKHAFAJI, S.R.; FARID, M. An investigation on pulsed electric fields technology using new treatment chamber design. Innovative Food Science and Emerging Technologies, v.8, n.2, p.205-212, 2007. Disponível em: <http:// www.sciencedirect.com/science/article/pi i/ S1466856406000865>. Acesso em: 20 nov. 2010. doi:10.1016/ j.ifset.2006.11.001.

ARONSSON, K. et al. Inactivation of Escherichia coli, Listeria innocua and Saccharomyces cerevisiae in relation to membrane permeabilization and subsequent leakage of intracellular compounds due to pulsed electric field processing. International Journal of Food Microbiology, v.99, p.1932, 2005. Disponível em: <http://www.sciencedirect.com/ science/article/pii/S0168160504003988>. Acesso em: 15 nov. 2009. doi:10.1016/j.ijfoodmicro.2004.07.012.

AZERÊDO, G.A. et al. Pulsos elétricos na conservação de alimentos: fatores críticos na inativação microbiana e efeitos sobre os constituintes alimentares. Boletim do CEPPA, v.26, n.2, p.171-178, 2008.

BARBOSA-CÁNOVAS, G.V et al. Conservación no térmica de alimentos. Zaragosa: Acribia, 1999. 280p.
BAZHAL, M.I. et al. Pulsed field treatment of apple tissue during compression for juice extraction. Journal of Food Engineering, v.50, p.129-139, 2001. Disponível em: <http:/ / www.sciencedirect.com/science/article/pii/ S0260877400002351>. Acesso em: 05 jan. 2011. doi:10.1016/ S0260-8774(00)00235-1.

BENDICHO, S. et al. Milk processing by high intensity pulsed electric fields. Food Science and Technology, v.13, p.195204, 2002a. Disponível em: <http://journals.cambridge.org/ action $/$ display Abstract fromPage $=$ online $\&$ aid $=109021>$. Acesso em: 20 jan. 2011. doi: DOI: 10.1017/ S0022029901005258

BENDICHO, S. et al. Effect of high intensity pulsed electric fields and heat treatments on vitamins of milk. Journal of Dairy Research, v.69, p.113-123, 2002b. Disponível em: < http:// journals.cambridge.org/abstract_S0022029901005258>. Acesso em: 20 jan. 2011. doi:10.1016/S0924-2244(02)00132-2.

BEVERIDGE, J. R. et al. The influence of pulsed duration on the inactivation of bacteria using monopolar and bipolar profile pulsed electric fields. IEEE Transactions on Plasma Science, v.33, n.4, p.1287-1293, 2005. Disponível em: <http:// ieeexplore.ieee.org/stamp/stamp.jsp?tp=\&arnumber= 1495571>. Acesso em: 20 mar. 2010. doi: 10.1109/ TPS.2005.852345.

CORTÉS, C. et al. Color of orange juice treated by high intensity pulsed electric fields during refrigerated storage and comparison with pasteurized juice. Food Control, v.19, p.151-158, 2008. Disponível em: <http://www.sciencedirect.com/science/article/ pii/S0956713507000503>. Acesso em: 20 mar. 2010. doi:10.1016/j.foodcont.2007.03.001

CRAVEN, H.M. et al. Evaluation of pulsed electric field and minimal heat treatments for inactivation of Pseudomonas and enhancement of milk shelf-life. Innovative Food Science and Emerging Technologies, v.9, p.211-216, 2008. Disponível em: <http://www.sciencedirect.com/science/article/ pii/S1466856407001671>. Acesso em: 30 ago. 2009. doi:10.1016/j.ifset.2007.11.002.

CSERHALMI, Z. et al. Study of pulsed electric field treated citrus juices. Innovative Food Science and Emerging Technologies, v.7, p.49-54, 2006. Disponível em: <http:// www.sciencedirect.com/science/article/pii/ S1466856405001086>. Acesso em: 20 mar. 2010. doi:10.1016/ j.ifset.2005.07.001.

DEVLIEGHERE, F. et al. New preservation technologies: Possibilities and Limitations. International Dairy Journal, v.14, p.273-285, 2004. Disponível em: <http:// www.sciencedirect.com/science/article/pii/ S0958694603001754>. Acesso em: 20 mar. 2010. doi:10.1016/ j.idairyj.2003.07.002.

ELEZ-MARTíNEZ, P. et al. Comparative study on shelf life of orange juice processed by high-intensity pulsed electric fields or heat treatment. European Food Research Technology, v.222, p.321-329, 2006. Disponível em: <http:// www.springerlink.com/content $/ \mathrm{j} 0522417057 \mathrm{r} 871 \mathrm{j} />$. Acesso em: 30 set. 2010. doi: 10.1007/s00217-005-0073-3.

EVRENDILEK, G.A.; ZHANG, Q.H. Effects of pulse polarity and pulse delaying time on pulsed electric fields-induced 
pasteurization of $\boldsymbol{E}$. Coli O157:H7. Journal of Food Engineering, v.68, n.2, p.271-276, 2005. Disponível em: $<$ http://www.sciencedirect.com/science/article/pii/ S0260877404002833>. Acesso em: 25 mar. 2010. doi:10.1016/ j.jfoodeng.2004.06.001.

GARCIA, D. et al. Bacterial resistance after pulsed electric fields depending on the treatment medium $\mathrm{pH}$. Innovative Food Science and Emerging Technologies, v.6, n.4, p.388395, 2005. Disponível em: <http://www.sciencedirect.com/ science/article/pii/S146685640500055X>. Acesso em: 20 mar. 2010. doi:10.1016/j.ifset.2005.04.003.

GARCIA, D. et al. Pulsed electric fields cause bacterial envelopes permeabilization depending on the treatment intensity, the treatment medium $\mathrm{ph}$ and the microorganism investigated. International Journal of Food Microbiology, v.113, n.2, p.219-227, 2007. Disponível em: <http:// ww w.s ciencedirect.com/s cience/article/pi i/ S0168160506004260>. Acesso em: 15 ago. 2009. doi:10.1016/ j.ijfoodmicro.2006.07.007.

HO, S.Y. et al. Effects of high field electric pulses on the activity or selected enzymes. Journal of Food Engineering, v.31, p.69-84, 1997. Disponível em: <http:// www.sciencedirect.com/science/article/pi i/ S0260877496000520>. Acesso em: 20 mar. 2010. doi:10.1016/ S0260-8774(96)00052-0.

KNORR, D. et al. Processing concepts based on high intensity electric field pulses. Trends in Food Science \& Technology, v.12, p.129-135, 2001. Disponível em: <http:// w w w. sciencedirect.com/science/article/pi i/ S0924224401000693>. Acesso em: 25 mar. 2010. doi:10.1016/ S0924-2244(01)00069-3.

LEBOVKA, N. et al. On the origin of the deviation from the first-order kinetics in inactivation of microbial cells by pulsed electric fields. International Journal of Food Microbiology, v.91, p.83-89, 2004. Disponível em: <http:// www.sciencedirect.com/science/article/pi i/ S0168160503003210>. Acesso em: 05 mar. 2009.

LI, S.Q.; ZHANG, Q.H. Inactivation of $\boldsymbol{E}$. coli 8739 in enriched soymilk using pulsed electric fields. Journal of Food Science, v.69, n.7, p.169-174, 2004. Disponível em: <http:/ / onlinelibrary.wiley.com/doi/10.1111/j.13652621.2004.tb13616.x/abstract>. Acesso em: 20 mar. 2010. doi: $10.1111 / \mathrm{j} .1365-2621.2004 . t b 13616 . x$.

LI, Y.Q. et al. Inactivation of soybean lipoxygenase in soymilk by pulsed electric fields. Food Chemistry, v.109, p.408-414, 2008. Disponível em: <http://www.sciencedirect.com/science/ article/pii/S0308814608000204>. Acesso em: 06 mar. 2009. doi: $10.1016 /$ j.foodchem.2008.01.001.

LÓPEZ, N. et al. Effects of pulsed electric fields on the extraction of phenolic compounds during the fermentation of must of tempranillo grapes. Innovative Food Science and Emerging Technologies, v.9, p.477-482, 2008. Disponível em: <http://www.sciencedirect.com/science/article/pii/ $\mathrm{S} 146685640700166 \mathrm{X}>$. Acesso em: 20 mar. 2010. doi:10.1016/j.ifset.2007.11.001.

MOSQUEDA-MELGAR, J. et al. Combination of high-intensity pulsed electric fields with natural antimicrobials to inactivate pathogenic microorganisms and extend the shelf-life of melon and watermelon juices. Food Microbiology, v.25; p.479-491, 2008. Disponível em: <http://www.sciencedirect.com/science/ article/pii/S0740002008000191>. Acesso em: 07 mar. 2010. doi:10.1016/j.fm.2008.01.002.

NIELSEN, H.B. et al. Consumer perception of the use of highpressure processing and pulsed electric field technologies in food production. Appetite, v.52, p.115-126, 2009. Disponível em: <http://www.sciencedirect.com/science/article/pii/ S0195666308005540>. Acesso em: 20 mar. 2010. doi:10.1016/ j.appet.2008.09.010

ODRIOZOLA-SERRANO, I. et al. Impact of high-intensity pulsed electric fields variables on vitamin $\mathrm{C}$, anthocyanins and antioxidant capacity of strawberry juice. LWT - Food Science and Technology. v.42, p.93-100, 2009. Disponível em: <http:/ / ww w.sciencedirect.com/s cience/article/pi i/ S002364380800128X>. Acesso em: 05 jan. 2010. doi:10.1016/ j.lwt.2008.05.008.

OTUNOLA, A. et al. Effectiveness of pulsed electric fields in controlling microbial growth in milk. International Journal of Food Engineering, v.4, p.1-14, 2008. Disponível em: $<\mathrm{http}$ ://www.bepress.com/ijfe/vol4/iss7/art1/>. Acesso em: 20 mar. 2010. doi: 10.2202/1556-3758.1494.

RAMOS, A.M. et al. Aplicação de campos elétricos pulsados de alta intensidade na conservação de alimentos. Ceres, v.53; n.308, p.425-438, 2006

RIENER, J. et al. Combined effect of temperature and pulsed electric fields on soya milk lipoxygenase inactivation. European Food Research and Technology, v.227, p.14611465, 2008. Disponível em: <http:/www.springerlink.com/ content/p347246647011183/>. Acesso em: 09 jan. 2010. doi: 10.1007/s00217-008-0868-0.

SAMPEDRO, F. et al. Pulsed electric fields inactivation of Lactobacillus plantarum in an orange juice-milk based beverage: effect of process parameters. Journal of Food Engineering, v.80, p.931-938, 2007. Disponível em: <http:// www.sciencedirect.com/science/article/pi i/ S0260877406005474>. doi:10.1016/j.jfoodeng.2006.08.013.

SOLIVA-FORTUNY, R. et al. Effects of pulsed electric fields on bioactive compounds in foods: a review. Trends in Food Science \& Technology, v.20, p.544-556, 2009. Disponível em: <http://www.sciencedirect.com/science/article/pii/ S0924224409002258>. Acesso em: 20 mar. 2010. doi:10.1016/ j.tifs.2009.07.003.

TEIXEIRA, L.J.Q. Aplicação de campos elétricos pulsados de alta intensidade no processamento de suco de cenoura. 2008. 168f. Tese (Doutorado em Ciência em Tecnologia de Alimentos) - Departamento de Ciência e Tecnologia de Alimentos. Universidade Federal de Viçosa, MG.

TEIXEIRA, L.J.Q. et al. Inactivation of oxidative enzymes by high-intensity pulsed electric field for retention of color in carrot juice. Food Bioprocess Technology, v.1, p.364-373, 2008. Disponível em: <http://www.springerlink.com/content/ 92g32p312840455g/>. Acesso em: 05 mar. 2010. doi: 10.1007/ s1 1947-007-0018-x.

WAN, J. et al. Advances in innovative processing technologies for microbial inactivation and enhancement of food safety $\mathrm{e}$ pulsed electric field and low-temperature plasma. Trends in 
Food Science \& Technology, v.20, p.414-424, 2009. Disponível em: <http://www.sciencedirect.com/science/article/ pii/S0924224409000521>. Acesso em: 05 out. 2010 doi:10.1016/j.tifs.2009.01.050.

ZHANG, Q.H. et al. Engineering aspects of pulsed eletric field pasteurization. Journal of food Engennering, v.25, p.261291, 1995. Disponível em: <http://www.sciencedirect.com/ science/article/pii/026087749400030D >. Acesso em: 24 set.2009. doi:10.1016/0260-8774(94)00030-D.

ZHAO, W.; YANG, R. Effect of high-intensity pulsed electric fields on the activity, conformation and self-aggregation of pepsin. Food Chemistry, v.114, p.777-781, 2009. Disponível em: <http://www.sciencedirect.com/science/article/pii/ S0308814608012235>. Acesso em: 23 set.2009. doi:10.1016 j.foodchem.2008.10.016.

ZHONG, K. et al. Inativation and conformational change of horseradish peroxidase induced by pulsed electric field. Food Chemistry. v.92, n.9, p.473-479, 2005. Disponível em: $<$ http://www.sciencedirect.com/science/article/pii/
S0308814604006247>. Acesso em: 21 mar. 2010. doi:10.1016/ j.foodchem.2004.08.010.

ZHONG, K. et al. Inactivation kinetics and secondary structural change of PEF-treated POD and PPO. Food Chemistry, v.100, p.115-123, 2007. Disponível em: <http:// www sciencedirect com/s cience/article/pii/ S0308814605008071>. Acesso em: 21 mar. 2010. doi:10.1016 j.foodchem.2005.09.035.

ZULUETA, A. et al. Ascorbic acid in orange juice-milk beverage treated by high intensity pulsed electric fields and its stability during storage. Innovative Food Science and Emerging Technologies, v.11, p.84-90, 2009. Disponível em: <http:// ww w. sciencedirect.com/science/article/pi i/ S1466856409000770>. Acesso em: 20 mar. 2010. doi:10.1016/ j.ifset.2009.07.007.

ZULUETA, A. et al. Fatty acid profile changes during orange juice-milk beverage processing by high-pulsed electric field. European Journal of Lipid Science and Technology, v.109, p.25-31, 2007. Disponível em: $<$ http://onlinelibrary.wiley.com/ doi/10.1002>. Acesso em: 20 mar. 2010. doi:10.1002/ ejlt.200600202. 